REVESCO. Revista de Estudios Cooperativos

ISSN: $1885-8031$

http://dx.doi.org/10.5209/REVE.59771

\title{
Responsabilidad social empresarial en banca. Su aplicación al caso de la banca cooperativa
}

\author{
Joan Ramón Sanchis Palacio ${ }^{1}$ y Sergio Rodríguez Pérez ${ }^{2}$
}

Recibido: 3 de diciembre de 2017 / Aceptado: 15 de enero de 2018

Resumen. El concepto de RSE y las dimensiones que lo componen vienen determinados por las características particulares de las empresas en cada industria o sector económico en el que operan. Esto es así por cuanto la definición de la RSE y su aplicación está condicionada a la operatividad, medición e implantación en el contexto en el cual se lleva a cabo. Por este motivo, para analizar el comportamiento de la RSE en la banca cooperativa, que es el objetivo del presente trabajo, es importante realizar un estudio sobre las implicaciones y las particularidades de ésta en el sector bancario, es decir, en el conjunto de las denominadas entidades de crédito. Los bancos cooperativos, más conocidos en España como cooperativas de crédito, han desempeñado tradicionalmente una destacada labor en el campo de la RSE, por su carácter de banca de proximidad (arraigada al territorio) y de empresa cooperativa (enfocada a las personas y a la comunidad). Aunque su peso dentro del sector bancario ha sido poco significativo (entre el 7\% y el 5\% según depósitos y créditos respectivamente), han desempeñado una función clave en la financiación de la economía productiva, en especial en sectores con mayores dificultades de acceso al crédito como el sector rural y las pequeñas y medianas empresas. Por estos motivos se ha considerado oportuno analizar la aplicación de la RSE al caso concreto de este tipo de organizaciones. Se ha realizado un análisis cualitativo, con el propósito de destacar en qué términos concretos la banca en general y la banca cooperativa en particular enfocan su actividad económica a la RSE y cómo lo hacen.

Palabras clave: Sostenibilidad; Sector bancario; Ética bancaria; Cooperativas de crédito; Inclusión financiera.

Claves Econlit: G21; M14; M21.

[en] Corporate social responsibility in banking. Its application to the case of the cooperative bank

Abstract. The concept of CSR and its dimensions depend on the particular characteristics of the economic sector in which companies operate. This means that the definition of CSR and its application is conditioned on how it works, is measured and implemented in the context in which it takes action. For this reason, to analyze the behavior of CSR in cooperative banking, which is the objective of this paper, it is essential to achieve a study on the implications and particularities of CSR in the banking sector, that is, in the whole of denominated credit institutions. The cooperative banks, better known in Spain as cooperatives credit, have traditionally played a prominent role in the field of CSR, due to their proximity banking status (rooted in the territory) and cooperative enterprise

1 Universitat de València, España

Dirección de correo electrónico: joan.r.sanchis@uv.es.

2 Universitat de València, España

Dirección de correo electrónico: serope@alumni.uv.es. 
(focused on people and the community). Although its weight in the banking sector has not been very significant (between 7\% and 5\% according to deposits and loans, respectively), they have played a key role in financing the productive economy, especially in sectors with higher access difficulties to credit, such as the rural sector and small and medium-sized enterprises. For these reasons, it has been considered appropriate to analyze the application of CSR to the particular case of this type of organization. A qualitative analysis has been carried out, with the aim of highlighting the specific terms the banking sector in general and cooperative banking, in particular, focus their economic activity on CSR and how they do it.

Keywords: Sostenibility; Banking sector; Banking ethic; Credit Cooperatives; Financial inclusion.

Sumario. 1. Introducción. 2. La RSE: concepto y dimensiones clave. 3. La RSE desde la perspectiva de las entidades de crédito. 4. La Relación entre la Banca Cooperativa y la RSE. 5. Conclusiones. 6. Referencias bibliográficas.

Cómo citar: Sanchis Palacio, J.R. y Rodríguez Pérez, S. (2018) Responsabilidad social empresarial en banca. Su aplicación al caso de la banca cooperativa. REVESCO. Revista de Estudios Cooperativos, Primer Cuatrimestre, No 127, pp. 204-227. DOI: 10.5209/REVE.59771.

\section{Introducción}

La reputación de la banca en España presenta uno de los peores niveles en Europa, situándose a la cabeza de los países con peor reputación, solo superada por la banca irlandesa. La Junta Europea de Riesgo Sistémico (European Systemic Risk Board), en su último informe de junio de 2015 basado en un trabajo de campo dirigido a 33 mil clientes bancarios de 27 países distintos, muestra una más que preocupante caída en la imagen de los bancos españoles, que desciende de un $31 \%$ (que es el nivel de confianza que tenía en el año 2009, año en el que se inicia la crisis financiera) a un 16\% en 2014 (con un descenso de 15 puntos), solo superado por los bancos irlandeses con un nivel de confianza del 15\% (solo un punto menos de diferencia). Aunque es un sector tremendamente regulado y supervisado, sigue teniendo graves problemas éticos (corrupción, sobornos, blanqueo de dinero, manipulación de tipos de interés y tipos de cambio, paraísos fiscales, ...) y de gobernanza (conflictos de interés, falta de transparencia y confianza), que han desembocado en una crisis reputacional en un sector basado fundamentalmente en la confianza. Precisamente por ello, es uno de los sectores más preocupado desde el principio por mejorar su imagen a través de estrategias de responsabilidad social corporativa (De la Cuesta González, 2017).

No obstante, dentro del sector bancario español existen diferentes tipos de entidades, entre las cuales se encuentran las cooperativas de crédito o bancos cooperativos, que mantienen una reputación mucho mayor y que no se han visto afectados por la crisis. Son entidades que tradicionalmente han seguido un comportamiento ético y responsible, que podríamos calificar de ejemplar, en muchos casos. Por este motivo, la banca cooperativa es el referente a seguir dentro del sector bancario en la aplicación de los principios de la ética y la responsabilidad social empresarial.

El presente trabajo tiene como objetivo analizar las características de la banca cooperativa como entidades pioneras y ejemplares en el uso de la responsabilidad social, comparándola con el resto de entidades del sector bancario español y con 
los diferentes elementos que definen el concepto de Responsabilidad Social Empresarial. Los trabajos publicados en este sentido son realmente escasos, por lo que nuestro estudio aporta una novedad al respecto y pretende ser el punto de partida, a través de un análisis meramente descriptivo, para la realización de un estudio posterior en mayor profundidad sobre el tema.

El trabajo se ha estructurado en cuatro apartados, además de este primero de introducción. En primer lugar se define el concepto de la Responsabilidad Social Empresarial (RSE) y se describen sus dimensiones clave. En segundo lugar se analiza la RSE desde el punto de vista de las entidades de crédito, para en tercer lugar aplicarlo al caso concreto de la banca cooperativa. El trabajo finaliza con unas conclusiones y reflexiones acerca del futuro de la RSE en la banca.

\section{La RSE: concepto y dimensiones clave}

La Responsabilidad Social Empresarial (RSE) es un concepto amplio y con unos límites muy difusos, formado por múltiples elementos y dimensiones, y al que se puede definir y acceder desde diversas perspectivas (Carroll, 1979).

Existen múltiples aportaciones de diferentes autores con propuestas para clasificar las diferentes teorías o enfoques en torno a la RSE. La realizada por Secchi (2007) plantea tres categorías de teorías: Utilitarias, Directivas y Relacionales. Otra aportación a destacar es la realizada por Reyes (2008), quien distingue cuatro diferentes tipos de estudios académicos: trabajos de concepto, propuestas que elaboran modelos generales o específicos que tratan de aplicar el concepto en las empresas, estudios que analizan la relación existente entre la implantación y desarrollo de políticas socialmente responsables y los resultados empresariales y trabajos que presentan la RSE como un caso de estudio o que analizan un caso referente a ésta en un sector específico. En nuestro caso, para tratar de afrontar esa diversidad, vamos a apoyarnos en la clasificación de los grupos de teorías que proponen Garriga y Melé (2004), que es asiduamente recurrida por la literatura y autores especializados en la materia, y que aporta cierta simplicidad y calidad explicativa al problema.

Estos autores, basándose en las teorías más relevantes, proponen cuatro categorías: teorías instrumentales, teorías políticas, teorías integrativas y teorías éticas. Las teorías instrumentales se basan en los aspectos más económicos de la RSE, y su objetivo es trazar estrategias que contribuyan a sacar el máximo beneficio para la sociedad derivadas de las acciones sociales que realiza la organización, tanto en términos de resultados como en forma de ventajas competitivas y en reputación social. Existen tres teorías principales en este grupo, dependiendo del objetivo económico perseguido: 1) las que se basan en diseños de estrategias que permitan a la organización conseguir ventajas competitivas que repercutan en mayores beneficios a largo plazo (Porter y Kramer, 2002); 2) los preocupados en maximizar el valor de la inversión realizada por el accionista y, por ende, el precio de la acción (Jensen, 2002); y 3) las relacionadas con el marketing como causa, en la que la RSE se convierte en una estrategia social que permite mejorar la imagen corporativa (Porter y Kramer, 2011).

Las teorías políticas hacen referencia a las interacciones y conexiones entre las empresas y la sociedad y en el poder y la posición de las empresas y su inherente 
responsabilidad (Garriga y Melé, 2004). En este grupo de teorías, los autores también distinguen tres subcategorías: 1) Constitucionalismo corporativo: existen causas tanto a nivel interno como externo que generan este poder (Davis, 1967); 2) Teoría integradora del contrato social: existen dos niveles de consenso, uno a nivel macro social, de carácter normativo, basado en hipernormas éticas y otro de carácter microsocial, que se refiere a los acuerdos implícitos o explícitos que tienen lugar entre los miembros de una comunidad específica y son los que generan las normas auténticas (Donaldson y Dunfee, 1994); y 3) Ciudadanía corporativa: es una nueva conceptualización del papel de la empresa en la sociedad, que introduce como hipernorma la declaración universal de los derechos humanos (Logsdon y Wood, 2002).

Las teorías integrativas pretenden incorporar en las empresas las demandas sociales, bajo el argumento de que las empresas dependen de la sociedad para su existencia y que, por tanto, es necesario si se quiere garantizar el desarrollo de ésta, hacerse eco de las reivindicaciones sociales e integrarlas en las organizaciones. De esta manera, la empresa estará actuando de acuerdo con los valores de sus grupos de interés y adquirirá de esta forma prestigio social. Estas teorías se subdividen, a su vez, en cuatro subcategorías: aspectos administrativos o de gestión, principio de responsabilidad pública, gestión de grupos de interés y desempeño social corporativo (Carroll, 1979).

Por último, las teorías éticas incluyen aquellas que "se basan en los requerimientos éticos que cimientan las relaciones entre las empresas y la sociedad" (Garriga y Melé, 2004: 60). Por lo tanto, entienden como necesario la aceptación de responsabilidades en la sociedad como parte de las obligaciones éticas de la empresa y se fundamentan en principios que expresan qué se debe hacer y qué no se debe hacer o la necesidad de construir una sociedad mejor. Estas estarían compuestas por cuatro subclases: teoría normativa de los grupos de interés, derechos humanos, desarrollo sostenible y enfoque del bien común (Freeman, 1994; Melé, 2002).

Teniendo en cuenta la variedad de enfoques y clasificaciones teóricas que existen del término RSE, era de esperar que existieran diferentes modelos que expliquen las distintas dimensiones que el marco conceptual abarca (García-Torea et al., 2017).

Aunque se puede encontrar un gran número de trabajos que proponen una RSE desde una escala unidimensional de medida (Marín y Ruiz, 2007), los modelos que hoy en día están más reconocidos en el ámbito académico coinciden en su carácter multidimensional, aún a pesar de que también existen numerosas diferencias entre ellos. Como más destacadas, queremos señalar tres de estas corrientes: la pirámide de Carroll $(1979,1999)$, los centrados en la Teoría de los grupos de interés (Maignan et al., 1999; Decker, 2004; Turker, 2009; Pérez y Rodríguez del Bosque, 2012) y los basados en la Teoría del desarrollo sostenible (Van Marrewijk, 2003; Panwar et al., 2006).

Desarrollada por Archie Carroll en 1979, y actualizada por él mismo en 1991 y 1999, la pirámide de Carroll trata de explicar la RSE a través de una pirámide con diferentes niveles que vienen a representar las diferentes dimensiones que están involucradas en este concepto, al comprender las diferentes expectativas que la sociedad tiene de las organizaciones en un momento dado, siendo éstas 
económicas, legales, éticas y voluntarias o filantrópicas, y estando todas ellas interrelacionadas entre sí. A pesar de tratarse de una aportación de gran reconocimiento durante mucho tiempo en el mundo académico, han surgido diferentes estudios que cuestionan su validez, poniéndose en duda que se reflejen de forma correcta la percepción de algún grupo de interés tan relevante como los consumidores (García de los Salmones et al., 2005).

Una perspectiva más actual para explicar los componentes de la RSE parte del enfoque de los grupos de interés (Freeman, 1994), proponiendo una dimensionalización del concepto a partir de los principales públicos objetivos que conforman la red de relaciones de la organización. Según esta propuesta, las acciones de RSE han de clasificarse preferentemente en función de aquellos grupos de interés que más se benefician con las mismas, y que son el público objetivo principal al que se quiere satisfacer con cada una de ellas (Martinez Garcia de Leaniz, 2015). Por lo tanto, teniendo en cuenta estos principios, los diferentes trabajos académicos han ido identificando distintas dimensiones para la RSE: consumidores, empleados, accionistas, sociedad general, medio ambiente y mercado, entre otros (Maignan et al., 1999; Decker, 2004; Turker, 2009; Pérez y Rodríguez del Bosque, 2011). Sin embargo, este modelo también es fuertemente cuestionado, ya que para algunos autores como Turker (2009), en este tipo de modelos no se logra abarcar a todos los verdaderos grupos de interés de la empresa, lo que limita considerablemente su capacidad como modelo.

Por último, el modelo Triple Bottom Line TBL, que es también conocido como modelo de desarrollo sostenible, triple balance o triple cuenta de resultado, incluye tres dimensiones diferentes: económica, social y ambiental, y al igual que en el modelo de Carroll, se defiende la interrelación entre ellas. Fue utilizado por primera vez por el fundador de la consultora Sustainability, John Elkington, en el libro publicado en 1997 Cannibals with Forks: The Triple Bottom Line of 21st Century Business (Elkington, 1997). El éxito de este modelo, que ha ido en crecimiento por su acogida en la implementación de políticas de RSE en las empresas, puede ser explicado entre otras cosas por dos razones importantes: 1) fácil comprensión de las dimensiones del modelo en objetivos empresariales (Linnanen y Panapanaan, 2002; Panwar et al., 2006); y 2) es la utilizada por el Global Reporting Initiative GRI para desarrollar guías para la elaboración de informes o memorias de sostenibilidad, que aporta una información corporativa comparable fundamentada en la implementación del TBL.

El concepto de RSE y las dimensiones que lo componen vienen determinados por las características particulares de las empresas en cada industria o sector económico en el que operan (Decker, 2004). Esto es así por cuanto la definición de la RSE y su aplicación está condicionada a la operatividad, medición e implantación en el contexto en el cual se lleva a cabo. Por este motivo, para analizar el comportamiento de la RSE en la banca cooperativa, que es el objetivo del presente trabajo, es importante realizar un estudio sobre las implicaciones y las particularidades de ésta en el sector bancario, es decir, en el conjunto de las denominadas entidades de crédito ${ }^{3}$. Los bancos cooperativos, más conocidos en

3 En nuestro análisis, nos centraremos atendiendo a las clasificaciones empleadas por GRI (www.globalreporting.org) en el segmento de bancos privados (banca comercial y de empresa), ya que es al que se corresponde el tipo de entidad que será objeto de nuestro análisis. 
España como cooperativas de crédito, han desempeñado tradicionalmente una destacada labor en el campo de la RSE, por su carácter de banca de proximidad (arraigada al territorio) y de empresa cooperativa (enfocada a las personas y a la comunidad) (Palomo Zurdo y Valor Martinez, 2001; Vargas Sanchez y Vaca Acosta, 2005). Aunque su peso dentro del sector bancario ha sido poco significativo (entre el $7 \%$ y el 5\% según depósitos y créditos respectivamente durante los últimos 10 años, según datos del Banco de España), han desempeñado una función clave en la financiación de la economía productiva, en especial en sectores con mayores dificultades de acceso al crédito como el sector rural y las pequeñas y medianas empresas. Por estos motivos se ha considerado oportuno analizar la aplicación de la RSE al caso concreto de este tipo de organizaciones. Se ha realizado de un análisis cualitativo, con el propósito de destacar en qué terminos concretos la banca en general y la banca cooperativa en particular enfocan su actividad económica a la RSE y cómo lo hacen.

\section{La RSE desde la perspectiva de las entidades de crédito}

La aplicación de la RSE al sector bancario se ha realizado desde temáticas y perspectivas diferentes, tal como se aprecia en los principales trabajos publicados, que quedan recogidos en la Tabla 1.

Tabla. 1. Enfoques temáticos de las investigaciones de RSE en el sector bancario

\begin{tabular}{|l|l|}
\hline Temática abordada & Autores \\
\hline Percepciones sobre RSE & $\begin{array}{l}\text { Chambers y Day (2009); Pérez y Rodríguez del } \\
\text { Bosque (2015). }\end{array}$ \\
\hline $\begin{array}{l}\text { Motivaciones en las } \\
\text { prácticas socialmente } \\
\text { responsables }\end{array}$ & $\begin{array}{l}\text { Polonsky y Scott (2005); McDonald y Rundle- } \\
\text { Thiele (2008); Senthikumar, Ananth y Arulraj } \\
(2011) .\end{array}$ \\
$\begin{array}{l}\text { Prácticas de RSE en el } \\
\text { sector bancario }\end{array}$ & $\begin{array}{l}\text { De la Cuesta González, Muñoz Torres y Fernández } \\
\text { Izquierdo (2006); Ruiz, Rios y Tirado (2009); } \\
\text { Seguí y Palomero (2013); Rice y Peter (2015). }\end{array}$ \\
\hline $\begin{array}{l}\text { Divulgación de la RSE en } \\
\text { el sector financiero }\end{array}$ & $\begin{array}{l}\text { Douglas, Doris y Johnson (2004); Maingot y } \\
\text { Zeghal (2008); Kahn, Halabi y Samy (2009); } \\
\text { Hassan (2009); Hassan y Syafri (2009). }\end{array}$ \\
\hline
\end{tabular}

Fuente: elaboración propia en base a Orozco et al. (2012)

Algunos autores destacan que el sector financiero es de los que más invierte a nivel mundial en RSE (Marín y Ruiz, 2007). Sin embargo, no es abundante la literatura existente que aborda la aplicación de la RSC en dicho sector (Truño i Gual, 2016; Pérez y Rodríguez Del Bosque, 2012), lo que refuerza nuestro propósito e interés en seguir analizando en este ámbito.

En cuanto al protagonismo del sector bancario en materia de RSE, conviene señalar que no se reduce solo al ámbito académico, sino que este sector es además pionero y ha ejercido una gran influencia en lo concerniente a la implantación, 
desarrollo e impulso de políticas de RSE y otras iniciativas relacionadas con la sostenibilidad. Este interés que hemos visto del sector bancario en la RSE, junto a ciertas características propias de la industria, acaban desembocando en una enorme repercusión en el desempeño de la RSE. Las causas que explican esta atracción e influencia son diversas, pero podemos agruparlas en tres principales: 1) $\mathrm{La}$ globalización, desregulación y aparición de nuevas tecnologías ha originado la sustitución de los tradicionales canales de distribución, de tal forma que los consumidores ya no distinguen diferencias sustanciales entre las distintas instituciones financieras (Gil et al., 2009). La RSE puede servir a las entidades de crédito como una herramienta que facilite su diferenciación; 2) La crisis económica de los mercados mundiales, salpicada de escándalos financieros (manipulación de índices de referencia, acuerdos para repartirse el mercado, etc.) y malas prácticas en el sector (colocación masiva de productos engañosos con riesgo, comisiones ilegales en la prestación de servicios, etc.) ha originado una pérdida total de confianza ${ }^{4}$ de la sociedad y un aumento de la conciencia social de reguladores, accionistas, socios, empleados y consumidores. La industria bancaria ha encontrado en la RSE un instrumento que puede ayudarles a restituir su imagen y dar respuesta de paso a esta creciente demanda de los agentes de una mayor transparencia y responsabilidad; y 3) el enorme protagonismo del sector en el impulso de la sostenibilidad se explica, en parte, por su papel central en el conjunto de la economía. Las entidades de crédito juegan un papel activo en el desarrollo económico y social de los países, ya que cuentan con la capacidad de seleccionar proyectos de inversión, gestionar riesgos y decidir quién accede al capital y qué actividades se financian (De La Cuesta González, 2006). Esta posición y rol dentro del sistema económico, facilita que la mayor contribución que puede realizar el sector a la RSE sea a través de su propio negocio, lo que conlleva unas responsabilidades implícitas. Este hecho ha generado cierto debate y presión en torno a las entidades, que conscientes de ello y de su potencial rentabilidad, ya han empezado a desarrollar desde hace años productos y servicios teniendo en cuenta criterios socialmente responsables (CECA, 2008).

Para analizar las singularidades de la RSE en las entidades bancarias, hay que distinguir una doble dimensión (Muñoz et al., 2004): la dimensión interna, referida a los impactos directos derivados de su comportamiento con relación a los diferentes grupos de interés, es decir, basados en mejorar la actuación de los servicios internos de los bancos; y la dimensión externa, sobre los impactos indirectos que resultan del papel que desempeñan los bancos al canalizar el ahorro hacia las actividades de inversión. Por tanto, el comportamiento de la RSE de las entidades bancarias debe juzgarse en las dos dimensiones de sus impactos (Cea Moure, 2010).

Según la última definición de 2011 sobre RSC de la UE, una entidad bancaria es socialmente responsable si en su actividad de inversión, gestión de activos e intermediación financiera gestiona adecuadamente los riesgos, oportunidades, e impactos que dicha actividad tiene la sociedad. Será socialmente responsable si se consideran cuestiones ambientales, éticas y sociales en el diseño de los productos

4 Según la EBA (Autoridad Bancaria Europea), desde el inicio de la crisis en 2008, el 97\% de los grandes bancos europeos ha estado involucrado en litigios por mala práctica bancaria, que les ha ocasionado el desembolso de más de cien millones de euros en compensaciones (Sanchis Palacio y Campos Climent, 2017). 
y servicios que oferta, en su política de crédito e inversión y en definitiva en toda su estrategia del negocio; así como si cuenta con mecanismos para controlarlo (sistemas de evaluación de impacto, incentivos y sanciones, transparencia y rendición de cuentas, etc) (Fernández Olit y De la Cuesta González, 2014). Su función social es ofertar productos necesarios y de calidad a los ciudadanos en función de sus necesidades y no solo de los intereses de la dirección de la entidad; algo que parece de sentido común pero que por desgracia no ha sido tan frecuente. Evitar ese riego legal de tener que compensar a los clientes por malas prácticas en el diseño y comercialización de productos no es solo una cuestión de interés general, sino de interés económico para la propia entidad, y es ahí donde reside la auténtica responsabilidad social de la banca, en hacer bien su negocio. Sin embargo, poca atención se ha prestado a esta visión comercial o de negocio de la RSC (De la Cuesta González, 2006) (De la Cuesta González et al., 2006; Esteban Sánchez et al., 2017).

Según De La Cuesta González (2017), la responsabilidad externa o comercial se prodía gestionar en base a los siguientes aspectos: 1) Aplicando criterios de sostenibilidad a la financiación y aseguramiento bancario (valoración previa a la concesión del crédito o garantía de los proyectos a financiar y/o asegurar bajo criterios de riesgo social, medioambiental); 2) Aplicando criterios de sostenibilidad a la gestión de activos y de inversión, fomentado lo que se denomina inversión socialmente responsable (ISR); y 3) Centrando un negocio banca comercial o minorista más responsable con el cliente, no solo desde el punto de vista de la calidad, transparencia y honestidad como señalan Pérez Ruiz y Rodríguez del Bosque (2012), sino poniendo el esfuerzo en conocer al consumidor y adaptar los productos, su coste y el canal de distribución a sus necesidades, mejorando de esta manera su calidad y accesibilidad.

Precisamente, la consecuencia de la acción en esta última dimensión externa es donde encontramos ciertos elementos que hacen distintiva la contribución que puede aportar la gestión de la RSE desde las entidades bancarias. A continuación, destacamos algunos de los más relevantes.

\subsection{Inclusión/Exclusión financiera}

La inclusión financiera es definida como "el acceso que tienen las personas y las empresas a una variedad de productos y servicios financieros útiles y asequibles que satisfacen sus necesidades" $\mathrm{y}$ es considerada por las autoridades normativas a nivel mundial, como una trascendental prioridad en materia de desarrollo. Así lo demuestra iniciativas surgidas durante la última década por organismos como el G20, que eligió el tema de la inclusión financiera como uno de sus pilares en la Cumbre de Pittsburgh de 2009 y decidió poner en marcha un grupo de expertos que se dedica exclusivamente a los temas de la inclusión financiera; o el Banco Mundial, que impulsó el programa UFA2020 para facilitar que las personas en todo el mundo tengan acceso a una cuenta para transacciones para finales de 2020.

Esta preocupación por la inclusión financiera es sustentada en la información arrojada por numerosos estudios (Banerjee et al., 2015), que proporcionan datos

5 Definición adoptada por el Banco Mundial, en www.bancomundial.org/es/financialinclusion. 
heterogéneos que sugieren que los mercados financieros incluyentes y eficientes son capaces de mejorar las condiciones de vida de los ciudadanos, reducir los costes de transacción, impulsar la actividad económica y mejorar la prestación de otros beneficios sociales. De todos estos estudios, el de Cull et al. (2014) clasifica los beneficios de la inclusión financiera bajo tres niveles diferentes: 1) a nivel microeconómico, el uso de diversos productos financieros incide en las condiciones de vida de las personas con menos recursos. Los productos de ahorro les permite gestionar los flujos de efectivo y ordenar el consumo (Brune et al., 2011), los seguros pueden ayudar a las familias a mitigar los riesgos y gestionar las crisis (Janzen y Carter, 2013) y ciertos tipos de servicios de pago pueden minorar los costes de transacción (Aker et al., 2011). Además, los estudios también demuestran que cuanto más inclusivo es el sistema, más opciones tienen las pequeñas empresas de beneficiarse del acceso al crédito, lo que genera inversión y repercute en el beneficio de la sociedad en la que se instalan; 2 ) a nivel de actividad económica local, algunas pruebas empíricas realizadas demuestran que el acceso financiero mejora la actividad económica local (Cull et al., 2014): las diferencias locales en la apertura de sucursales bancarias en zonas rurales no bancarizadas de la India (impulsada por exigencias del organismo regulador entre 1977 y 1990) estaban vinculadas con una reducción significativa de la pobreza rural (Burgess y Pande, 2005) y la rápida apertura de sucursales de Banco Azteca en México en más de un millar de tiendas minoristas del Grupo Elektra significó un impacto en los niveles generales de ingresos en la región del 7\% en comparación con comunidades similares en las que no se había abierto ninguna sucursal (Bruhn y Love, 2012); y 3) a nivel macroeconómico, las investigaciones sugieren la existencia de una correlación positiva entre la inclusión financiera y el crecimiento y el empleo. Los principales mecanismos que citan a ese respecto son: los costes de transacción generalmente más bajos, una mejor distribución del capital (Jahan y McDonald, 2011) y mayor estabilidad financiera y control de los riesgos en toda la economía (Han y Melecky, 2013).

El efecto opuesto a la inclusión financiera es la exclusión financiera, definida ésta por la Comisión Europea ${ }^{6}$ como "el proceso mediante el cual las personas se encuentran con dificultades en el acceso y/o usos de servicios financieros adecuados a sus necesidades y que les permita llevar una vida social normal en la sociedad a la que pertenecen". Las causas que lo motivan son diversas, y pueden estar asociadas a su ubicación geográfica (zonas marginadas, mal comunicadas, etc.) o a sus propias características como colectivo (personas de bajos ingresos, parados, inmigrantes, personas con escaso nivel de estudios, etc.). En cualquier caso, parece evidente que el interés por la inclusión financiera y la facilitación de acceso a servicios bancarios, tanto a particulares como a pymes, en colectivos que quedan fuera del sistema, consigue paliar esta exclusión financiera y la falta de oportunidades en diferentes ámbitos como en el mercado de la vivienda, empleo, salud, escolarización, ciudadanía, sobreendeudamiento, etc., y que son causa de mayor exclusión social (Kempson et al., 2000); siendo por tanto uno de los principales espacios de intervención de las entidades financieras en la sociedad (CECA, 2008). A través de la inclusión financiera, las entidades bancarias tienen

6 European Commision (2008): "Finantial services provision and prevention of financial exclusion". 
además de una oportunidad de negocio (incluir a más personas en su cartera de clientes), la mejor opción para incidir sobre las condiciones de vida de las poblaciones en las que operan, haciendo una contribución específica derivada de su naturaleza desde la RSE (Decker, 2004).

\subsection{Lucha contra el blanqueo de capitales y la corrupción}

El Fondo Monetario Internacional define el blanqueo de capitales como "el proceso mediante el cual se intenta disimular el origen delictivo de determinados fondos que son producto de una actividad encubierta" como son el narcotráfico, el contrabando, la trata de seres humanos o la corrupción. El blanqueo de capitales está establecido como un delito contra el orden económico y social, por la exposición permanente de las personas y empresas involucradas directa e indirectamente en esta amenaza. Las estimaciones del Fondo Monetario Internacional consideran que el volumen de capitales blanqueados cada año oscila entre el 3\% y el 5\% del Producto Bruto Mundial (FMI, 2016).

La utilización de diferentes servicios bancarios es una de las principales vías de blanqueo de capitales. La globalización de los mercados financieros, la complejidad para establecer herramientas de control a nivel mundial y la elaboración de productos cada vez más sofisticados, han favorecido la proliferación de una auténtica inteligencia financiera destinada a la ocultación del origen de capitales obtenidos por medios ilegales.

Las entidades de crédito figuran en el primer frente de esta lucha y están obligadas a aplicar ciertas medidas de prevención en todas aquellas circunstancias de riesgo: Identificación de sus clientes, comunicación de operaciones sospechosas, reporting sistemático, conservación de documentos y confidencialidad. Dado el papel fundamental que desarrollan las entidades financieras en la obstaculización del blanqueo de capitales, se han extendido diferentes iniciativas autoreguladoras para el propio sector financiero, como por ejemplo las desarrolladas por el grupo Wolfsberg (http://www.wolfsberg-principles.com/index.html), formado por trece bancos globales en el año 2000, y que se han ido revisando desde entonces, con el objetivo de desarrollar marcos y directrices que faciliten la gestión de delitos financieros como el blanqueo de capitales.

En definitiva, podemos decir que nos encontramos en un momento en el que los costes asociados al cumplimiento de la regulación contra el blanqueo de capitales por parte de las entidades bancarias han tenido un tremendo ascenso, y que el interés que le conceden los altos directivos está disminuyendo (Fernández et al., 2013). Por tanto, desde la perspectiva de la RSE, esta lucha puede significar para ellas una nueva oportunidad de mejorar significativamente los distintos entornos sociales en los que desarrollan sus actividades, lo que debería servir para incitarlas a tomar medidas que signifiquen una fuerte apuesta por la transparencia y el adecuado control de riesgos.

\subsection{Inversión Socialmente Responsable ISR}

La ISR se define como el proceso de inversión en el que en la toma de decisiones se tienen en cuenta además de los tradicionales criterios de seguridad, rentabilidad 
y liquidez, otros aspectos ambientales, sociales y de gobierno corporativo (criterios ASG). Es un vehículo impulsor de la RSE al estimular a las empresas a incorporar la gestión de su responsabilidad social, ética y ambiental en el núcleo del negocio, para contribuir en las tomas de decisiones de inversión. Desde las entidades bancarias, el uso de la ISR se hace desde sus dos dimensiones interna y externa ${ }^{7}$.

Desde la óptica interna, las consecuencias se derivan a través del diseño y oferta de un catálogo de productos y servicios que cumplan ciertos criterios comprometidos con la inversión responsable y que sirven para satisfacer las demandas y necesidades de sus diferentes grupos de interés (empleados, socios, clientes, accionistas...).

Desde la óptica de su dimensión externa, es la misma industria financiera quien decide dónde invertir los depósitos que recibe de sus clientes, por quien apostar y a quien financiar, teniendo presente en mayor o menor medida criterios ASG y condicionando el tipo de desarrollo de una región o país. Siendo la acción en ambas dimensiones muy deseables y compatibles, es desde esta última vertiente desde dónde el sector bancario tiene una mayor capacidad de generar impactos indirectos y desde donde se genera una contribución específica muy valiosa del sector a la RSE.

Este tipo de inversión considera imprescindible conocer el desempeño ambiental, social y de gobernanza de las empresas en las que invierte para garantizar una rentabilidad financiera del capital en el medio y largo plazo. El mayor volumen del patrimonio ISR se concentra en la inversión institucional (fondos de inversión, fondos de pensiones e incluso fondos soberanos) aunque cada vez más la inversión minorista reclama una gestión responsable de sus fondos y crece de forma importante la denominada inversión de impacto. También las estrategias de gestión han evolucionado pasando de aplicarse simples criterios de exclusión a integrarse estos criterios extrafinancieros en la gestión financiera habitual (Eurosif, 2016).

Recordamos, para finalizar, que posiblemente la ISR es el pilar de entre los que se fundamenta la RSE, que más influencia e impacto tiene en el sector bancario (Moneva y Lizcano, 2003), y otra gran oportunidad para esta industria de comprometerse a través de la financiación de la economía productiva y real en lugar de la especulativa y bajo criterios socialmente responsables.

En definitiva, se puede decir que la verdadera RSE de los bancos se encuentra en la dimensión externa y los impactos indirectos (económicos, sociales y ambientales) producidos por las actividades que deciden financiar (Rosero Villabon, 2015). También es en esta dimensión externa donde aparecen ciertos elementos, como la facilitación de la inclusión financiera, la lucha por el blanqueo de capitales y el desarrollo de la ISR, que hacen distintiva y especialmente valiosa la contribución que pueden aportar las entidades bancarias a la gestión de la RSE y el desarrollo sostenible. La mayor complejidad para medir estos impactos indirectos es quizás la explicación para que estos no cuenten con el protagonismo correspondiente en los informes de sostenibilidad publicados por las entidades bancarias. A pesar de ello, hay que reconocer que estas entidades cada día tienen más en cuenta criterios de ASG en el momento de seleccionar proyectos de 
financiación, lo que se traduce en impactos más socialmente responsables y deseados. La Tabla 2 resume los aspectos más relevantes de la RSE aplicada a las entidades bancarias.

Tabla. 2. Aspectos más relevantes de la RSE en el sector bancario

\begin{tabular}{|c|c|}
\hline Dimensión interna & Dimensión externa \\
\hline $\begin{array}{l}\text { Gestión ética de los suministros } \\
\text { oficinas }\end{array}$ & Inclusión financiera de calidad \\
\hline Oferta de productos éticos y solidarios & Lucha por el blanqueo de capitales \\
\hline $\begin{array}{l}\text { Calidad del puesto de trabajo e } \\
\text { igualdad }\end{array}$ & Desarrollo de la Inversión Responsable \\
\hline Reparto justo del volumen de trabajo & Negocio de banca comercial minorista \\
\hline $\begin{array}{l}\text { Promoción comportamiento ético en } \\
\text { trabajo }\end{array}$ & Sostenibilidad de la financiación \\
\hline $\begin{array}{l}\text { Reparto justo la renta (diferencias } \\
\text { salarios) }\end{array}$ & $\begin{array}{l}\text { Gestión responsable del riesgo de loa } \\
\text { activos }\end{array}$ \\
\hline \multirow[t]{3}{*}{ Democracia interna y transparencia } & $\begin{array}{l}\text { Sostenibilidad en el diseño de } \\
\text { productos }\end{array}$ \\
\hline & Transparencia y rendición de cuentas \\
\hline & Sistemas de evaluación del impacto \\
\hline
\end{tabular}

Fuente: elaboración propia

En definitiva, parece como las entidades bancarias cada vez son más conscientes de que las prácticas de responsabilidad social son una inversión con un impacto positivo sobre su desempeño, su reputación e imagen y, en definitiva, su sostenibilidad y creación de valor a largo plazo (Sarro Alvarez et al., 2007), lo que les ha llevado a convertirse en uno de los sectores de actividad pioneros en el desarrollo de iniciativas sectoriales y a la incorporación de políticas y prácticas de RSE. Todo esto unido a la influencia que es capaz de ejercer por su papel central en la economía y la capacidad de generar impactos a través de su dimensión externa, propia de su actividad de intermediación, convierte a las entidades bancarias en la industria probablemente con más interés y potencial en el ámbito de la sostenibilidad y la RSE.

\section{La Relación entre la Banca Cooperativa y la RSE}

La banca cooperativa, como entidad de crédito que es, comparte todas las contribuciones singulares que desde el sector bancario se hace a la RSE. Además, en alguna de ellas, como es la inclusión financiera, las cooperativas de crédito desarrollan un papel destacado y crucial, al ofrecer una inclusión financiera de calidad. Estas entidades bancarias, a diferencia de los bancos privados, han mantenido su negocio de banca minorista enfocada al cliente con criterios de sostenibilidad $\mathrm{y}$, por tanto, han conservado de manera casi intacta sus 
contribuciones a la RSE. No ha sido así en el resto de entidades bancarias (bancos y cajas de ahorros), que con el incremento de la concentración del sector y la trasnformación de la casi totalidad de las cajas en bancos, han abandonado en parte este enfoque de banca de proximidad, que las cooperativas de crédito en su mayoría aún conservan. En España se ha producido una reducción significativa del número de entidades bancarias, principalmente de las caracterizadas como banca de proximidad o community banking, lo que impide mantener el principio de diversidad financiera defendido por la Unión Europea.

Pero esta no es la única característica que las diferencias del resto de entidades del sector en su relación con la RSE. A continuación, tras realizar una revisión detallada de la literatura al respecto (Carrasco Monteagudo, 2005; Palomo Zurdo y Valor Martínez, 2001; Ontiveros y Sánchez, 2014), se realiza una exposición de los elementos intrínsecos en la naturaleza de las cooperativas de crédito que a nuestro criterio más la acercan a ciertos postulados de la RSE, y que las diferencian sobre el resto de la industria.

\subsection{Finalidad y modelo de negocio}

El origen de las cooperativas de crédito fue responder a las necesidades financieras de las clases sociales excluidas del mercado. Se adoptó el modelo cooperativo porque era el más apropiado para servir a este deseo, lo que las ha dotado de ciertas características distintivas: una clara orientación hacia los mercados locales/nacionales, la facilitación de la accesibilidad geográfica para sus socios y un margen de intervención estratégica en manos de los decisores locales (Malo y Tremblay, 2004).

Este modelo de negocio también conocido como Community Banking (banca de proximidad en España), tienen como principales actores, aunque no los únicos ${ }^{8}$, a las cooperativas de crédito. La Reserva Federal en EE.UU., por ejemplo, les dispensa un tratamiento especial y las define como "el segmento de aquellas instituciones de crédito y ahorro que tienen propietarios y desarrollan sus operaciones en el ámbito local. Entidades que se concentran en las necesidades de las comunidades donde tienen sus oficinas y cuyas decisiones de financiación se toman por ejecutivos que entienden perfectamente las necesidades de esas familias y pymes locales, y cuyos riesgos se gestionan por empleados que no solo tienen interés en el cliente, sino que son parte de las comunidades a las que sirven y con las que están profundamente comprometidos" (Ontiveros y Sánchez, 2014: 72).

Desde su origen, por tanto, las cooperativas de crédito han asumido responsabilidades sociales e integran en su estructura los intereses de las comunidades donde desarrollan su actividad. Esta internalización de la RSE por parte de las cooperativas de crédito se basa tanto en sus valores y principios específicos, como en la configuración empresarial del modelo cooperativo que mencionábamos y que implica que las diferentes partes interesadas estén

8 El Comité Económico y Social CESE de la Unión Europea incluye como banca de proximidad o community banking a las cooperativas de crédito y a las cajas de ahorros. Sin embargo, en España, las cajas de ahorros se han transformado en bancos privados según la Ley 26/2013, de 27 de diciembre, de cajas de ahorros y fundaciones bancarias privadas, quedando en la actualidad solamente dos: Caixa Ontinyent en la Comunidad Valenciana y Caixa Pollensa en Baleares. 
incorporadas estructuralmente, y no como sucede en el resto de modelos de empresas, donde su integración requiere que se diseñen fórmulas e instrumentos específicos (Buendia Martínez y Tremblay, 2015).

Una prueba de este reconocimiento a las cooperativas de crédito en el desarrollo ha sido puesta de manifiesto por la Unión Europea ${ }^{9}$, que considera que su función es clave en el desarrollo regional facilitando la integración y cohesión social, siendo especialmente relevante su aportación en áreas económicas débiles donde con la reinversión de los beneficios en los lugares de origen, contribuyen en la generación y el mantenimiento del empleo, de infraestructuras socioeconómicas diversas y de actividades encuadradas en el sector secundario y terciario (Belhouari et al., 2005).

También Naciones Unidas, desde una perspectiva más global, considera que las cooperativas de crédito son uno de los mejores exponentes para conseguir el desarrollo económico y social, al facilitar la satisfacción de las necesidades de servicios sociales básicos de una forma efectiva y razonable (United Nations, 2000).

\subsection{Arraigo territorial y desarrollo de la economía local}

Hasta ahora, las cooperativas de crédito se han caracterizado por su marcado carácter territorial (Melian et al., 2010). Además, han logrado conservar algunas peculiaridades, como una fuerte orientación hacia su mercado y una mayor accesibilidad geográfica y social que les facilita el mantenimiento de relaciones de proximidad con sus socios y sus comunidades. Esto les ha permitido como entidades de depósito, canalizar el ahorro local hacia proyectos que surgen de la comunidad donde operan, contribuyendo a reducir fugas de capital o el éxodo de personas hacia zonas más industrializadas.

Se puede decir, por tanto, que las cooperativas de crédito han contribuido de forma decisiva a la reducción de los efectos de la exclusión financiera en el ámbito rural y local, proporcionando productos y servicios financieros a las familias y a las pequeñas y medianas empresas en territorios deprimidos (Palomo Zurdo y Valor Martínez, 2001), y que favorecido por su estructura societaria y democrática, ha cumplido una importante labor en estimular el desarrollo de la economía real en el ámbito local y regional.

Un ejemplo práctico de como las cooperativas de crédito pueden contribuir al proceso del desarrollo local, se puede observar en la posibilidad que ofrece a los agentes tanto públicos como privados, de acceder a financiación privilegiada a través de dos posibles tipos de estrategias: 1) constituyendo una alianza con otros agentes locales con los que compartan objetivos e intereses para constituir una cooperativa de crédito; y 2) establecer una colaboración de diferentes actores locales interesados y una cooperativa de crédito ya existente, que les permita llegar a una serie de acuerdos preferenciales. Este tipo de iniciativas constituyen una alternativa a otras fuentes de financiación clásicas, y su implementación en la

9 En su "Resolución sobre la contribución de las cooperativas al desarrollo regional", Diario Oficial de las Comunidad Europeas (D.O.C.E.), n. C 61/231, del 11 de febrero 1994. 
actualidad, demuestran las enormes posibilidades que para el desarrollo local pueden suponer (Melián Navarro et al., 2010).

Por otra parte, se debe señalar que la globalización y la desregulación de los mercados financieros, en una sociedad que además es cada vez menos agraria, menos rural y en claro proceso de terciarización, está incrementando la presión sobre las cooperativas de crédito. Estas se están viendo forzadas a conseguir un redimensionamiento óptimo que se adapte a su propia idiosincrasia y a la búsqueda de nuevas oportunidades de negocio que les exige realizar una selección minuciosa de clientes y regiones donde desarrollar su actividad.

En este escenario, resulta imprescindible que los criterios de arraigo territorial, compromiso con la comunidad local y enfoque a las necesidades reales del contexto que les rodea sigan presente en la toma de decisiones. Esto les permitirá mantener esa importante labor de inclusión social y dinamización de la economía en el ámbito local que siempre han desempeñado.

\subsection{Gobierno corporativo}

Uno de los pilares que hacen posible la RSE es el buen gobierno corporativo. Este trata de optimizar la gestión de la organización a través de valores que implican una gestión más democrática y transparente, y el empleo de mecanismos de control que aseguren el correcto desempeño de sus funciones. Este modelo de buen gobierno corporativo es el que predomina en las sociedades cooperativas, las cuales por su naturaleza social desarrollan su actividad al servicio del socio y de la comunidad, bajo unas reglas de ética empresarial (Puentes Poyatos et al., 2009).

La naturaleza cooperativa y la vocación social de estas entidades, distinto del enfoque shareholder de las empresas cotizadas, plantean un punto de partida inmejorable para acometer políticas, medidas e instrumentos de RSE, así como desarrollar como hemos ya comentado uno de sus pilares fundamentales, el buen gobierno corporativo. Este gobierno corporativo, por tanto, presenta ciertas peculiaridades en el ámbito del cooperativismo de crédito, ya que el socio es la garantía y la correa de transmisión entre los valores y objetivos de la sociedad y la cooperativa de crédito (Cortes García y Belmonte Ureña, 2010).

Y aunque probablemente el crecimiento de algunas de estas entidades de crédito y la evolución del negocio financiero puede significar una amenaza que podría debilitar la cercanía con la que actúan y que las caracteriza, todavía se puede detectar cómo mantienen una especial vinculación con el entorno en el que se desarrollan, lo que les permite conservar valores que determinan un gobierno democrático y la capacidad de control por parte de los socios (Soler Tormo y Melián Navarro, 2012).

No obstante, a pesar de todo esto, el modelo cooperativo tiene espacio y potencial de mejora, en aspectos concernientes a la eficacia y eficiencia de su gobernanza, así como en el control para el adecuado desarrollo de su actividad. Concretamente, desde el sector de las cooperativas de crédito, su gobierno corporativo y enfoque hacia la RSE podría mejorarse si se incrementara la transparencia o se fomentara la información en torno a la participación de los stakeholders en la gestión de la cooperativa, comunicando, por ejemplo, la 
afluencia de los socios a la asamblea anual, o cómo participan los empleados y los socios en la gestión cotidiana de la cooperativa.

\subsection{Relación con los grupos de interés}

Previamente, ya se había destacado el hecho de que la naturaleza cooperativa de estas entidades contempla que el socio puede llegar a ejercer un rol múltiple (propietario, cliente, proveedor, trabajador, etc.), lo que propicia que existan ciertas particularidades en la relación con los diferentes grupos de interés, los que a diferencia de lo que ocurre en otro tipo de organización, son contemplados e incorporados estructuralmente, en el modelo de negocio.

La perspectiva más actual para explicar los componentes de la RSE parte del enfoque de los grupos de interés (Freeman, 1994), donde el objetivo estratégico de la empresa se amplía a la búsqueda de valor para todos los grupos de interés y no se limita a satisfacer únicamente al de los accionistas (teoría shareholder). Sin embargo, este enfoque de los grupos de interés no es nuevo para las sociedades cooperativas, sino que es un enfoque intrínseco a la naturaleza de éstas, las cuales tienen la capacidad para integrar las necesidades de los referidos grupos de interés y establecer con ellos sólidas relaciones basadas en la participación (Vargas Sánchez y Vaca Acosta, 2005).

Esto hecho, sin duda, favorece el desarrollo en estas organizaciones de la RSE, y las sitúa en una mejor posición estratégica tal y como reconoce la Comisión Europea (2002) al señalar que "Las cooperativas tienen una larga tradición en combinar viabilidad económica y responsabilidad social gracias al diálogo entre las partes interesadas y a la gestión participativa, y pueden servir de referencia a otras organizaciones".

\subsection{Fondos de reservas sociales: el FEP en España}

En el caso de España, existe la obligación de dotar una reserva específica destinada a financiar actividades de educación, formación y promoción de cooperativas y que afecta, por tanto, a las cooperativas de crédito. Dentro del ámbito europeo, esta obligación únicamente es exigida por las legislaciones del sur de Europa: además de España, en Italia y Portugal.

No obstante, en una gran cantidad de países es práctica común de las cooperativas y en concreto de las cooperativas de crédito, constituir fondos sociales con diferentes denominaciones, de carácter obligatorio o no, y de distintas dotaciones, pero que suelen tener en común la vocación social y el propósito de hacer cumplir los principios y valores sobre los que el modelo cooperativo se construyó.

A pesar de la falta de homogeneidad, consideramos que esta práctica no se debe pasar por alto en un análisis que relacione las particularidades de la banca cooperativa con la RSE y, por ello, desde el modelo español, vamos a detenernos brevemente en estudiar sus características, con la pretensión de que las conclusiones de este epígrafe sirvan tan solo como ejemplo y referencia y no como un marco generalizable y extrapolable de forma exhaustiva para el resto de bancos cooperativos. 
En España, el Fondo de Educación y Promoción (FEP) es una de las partidas más singulares y características de las sociedades cooperativas en general, siendo regulado tanto en lo que se refiere a su dotación ${ }^{10}$ como a su aplicación por la legislación cooperativa vigente. Se trata de un fondo obligatorio con la finalidad de que se apliquen a determinadas actividades que benefician a los socios, trabajadores y, en su caso, a la comunidad en general. Se puede emplear, entre otras muchas opciones, para promover la formación de socios y trabajadores, difundir y promocionar el cooperativismo, fomentar las relaciones intercooperativas, realizar acciones encaminadas a la conservación del medioambiente, etc. Su existencia forma parte de la propia razón de ser de estas entidades y su constitución se encuentra ligada a los principios cooperativos; en concreto, al tercero (Participación económica de los miembros), quinto (Educación, entrenamiento e información), sexto (Cooperación entre cooperativas) y séptimo principio (Compromiso con la comunidad) (Fundazioa, 2011).

Según Server Izquierdo y Villalonga Grañana (2008), en su estudio sobre el Fondo de educación y promoción como indicador social, se apunta que las tres principales partidas a las que se dedica el FEP son: la Promoción cultural, la difusión y promoción del cooperativismo y la formación y educación. También se señala en su trabajo que la partida destinada al medioambiente no ha contado con la misma atención por parte del cooperativismo de crédito.

También se destaca la circunstancia de que la dotación que hacen las cooperativas de crédito al FEP, fundamentalmente a partir del excedente neto, es superior al mínimo exigido en la ley en la gran mayoría de los casos, y que, junto al destino del mismo, nos permite mostrar la tendencia de estas entidades hacia conductas socialmente responsables y reconocer las aplicaciones de este fondo como una partida básica para la evaluación de la RSE (Server Izquierdo y Villalonga Grañana, 2008). Además, el hecho de que estén obligadas por ley a hacer de forma explícita acción social y, en concreto, al fomento del desarrollo económico y social en sus entornos regionales y locales, sirve para afirmar que la propia naturaleza de las cooperativas de crédito implica un comportamiento socialmente responsable (Server Izquierdo y Capó Vicedo, 2009).

\subsection{Principios cooperativos}

Como último elemento a destacar, entre los que sirven para caracterizar la especial vinculación entre la banca cooperativa y la RSE, hemos seleccionado los principios sobre los que se fundamenta el modelo cooperativo. Estos creemos pueden servir de síntesis de todo lo anteriormente expuesto, y la mejor forma de plasmar sus intrínsecos vínculos.

Los principios y valores son los elementos distintivos de las organizaciones y empresas cooperativas. En 1844, los Pioneros de Rochdale, fundadores de la primera cooperativa de la historia, habían formulado un sistema de principios simple y claro que les facilitaba la gestión de la organización en beneficio de sus miembros.

10 Según la legislación vigente, las cooperativas de crédito tienen que dotar obligatoriamente y como mínimo, un $10 \%$ de su excedente neto a este particular fondo. 
La II Asamblea General de la ACI, realizada en 1995 en la ciudad de Manchester, sirvió para realizar una renovada Declaración de Identidad Cooperativa que incluye una nueva definición de cooperativa y una revisión de la formulación de los Principios y Valores Cooperativos. Esta actualizada declaración respetó la esencia de un sistema de principios y valores que demostró ser eficiente durante tantas décadas de historia. De esta manera, la Alianza Cooperativa Internacional define la cooperativa "como una asociación autónoma de personas que se han unido de forma voluntaria para satisfacer sus necesidades y aspiraciones económicas, sociales y culturales en común mediante una empresa de propiedad conjunta y de gestión democrática" (ACI, 1995). En esta definición podemos entrever cuestiones relacionadas con la RSE, que quedan mucho más claras en la especificación de los siete Principios Cooperativos.

A diferencia de las sociedades cooperativas, que cuentan con estos principios y valores generalmente aceptados que orientan el funcionamiento de éstas, para las organizaciones socialmente responsables no existen unos principios formalmente establecidos y reconocidos que las delimiten. Los principios de RSE se derivan tanto de las definiciones de RSE más comúnmente aceptadas, como de las recomendaciones que al respecto hacen los organismos internacionales.

Los principios cooperativos, por tanto, están presentes a lo largo de todos los principios de RSE, siendo los principios de educación, formación e información, de cooperación entre cooperativas y de interés por la comunidad, los más presentes en esta relación. Esta relación viene a demostrar que los principios cooperativos podrían ser considerados como principios de RSE a seguir por las sociedades socialmente responsables. Por tanto, la aplicación de la RSE es consecuente con la profundización de algunos Principios Cooperativos, o visto de otra forma, que la aplicación rigurosa de los Principios Cooperativos está en línea con los mandatos de la RSE, convirtiéndose así las sociedades cooperativas en entidades modelos o exponentes de la RSE (Carrasco, 2005).

\section{Conclusiones}

Hemos destacado en apartados anteriores sobre el especial protagonismo que, dentro del sector de entidades de crédito, tiene la banca cooperativa en el ámbito de la inclusión financiera. Esto lo hemos podido observar reiteradamente a lo largo de los seis elementos que hemos señalado como elementos característicos de estas entidades cooperativas en su relación con la RSE y que las diferencias del resto de la industria.

Además, hemos podido entender, como su naturaleza cooperativa, finalidad, gobernanza, relación especial con los grupos de interés y los principios que inspiraron su constitución, explica por qué el concepto de RSE está tan estrechamente relacionado con el de la cooperación, concretamente en este caso, con el del crédito cooperativo, y que estas entidades, por tanto, están en la mejor posición para impulsar esta RSE.

No obstante, también hemos visto que según evoluciona el mercado (globalización, desregulación, terciarización, etc.) y estas entidades sufren procesos de redimensionamiento $y$ se diversifican territorialmente, van a tener que 
enfrentarse con retos como la menor participación de los socios, la mercantilización de su actividad bancaria y la baja actividad cooperativizada. Esto exigirá tomar medidas y realizar una revisión de los conceptos de democracia empresarial, de legitimidad y de representatividad de los órganos societarios, para que estas circunstancias no pongan en riesgo la efectiva implantación de la Responsabilidad Social Empresarial en este tipo de organizaciones.

La vocación social con la que fueron creadas, debe ser guía y fuente de inspiración para que puedan seguir realizando una contribución esencial en las comunidades sobre las que ejercen influencia, lo que redundará sin duda en una aportación beneficiosa a la sostenibilidad y al desarrollo de su RSE.

Además, las cooperativas de crédito, por su carácter de banca de proximidad y por las características descritas y resaltadas, pueden actuar de revulsivo de un sector, el bancario, que necesita urgentemente un verdado cambio de imagen. Aunque es cierto que el sector bancario en su conjunto ha desempeñado históricamente un papel destacado en la implementación de la RSE, también es verdad que sus malas prácticas y abusos, claramente visibilizadas durante los últimos 10 años, y en especial relacionadas con la crisis financiera de 2008, ha deteriorado de manera significativa su reputación e imagen corporativa. Por el contrario, las cooperativas de crédito no se han visto afectadas por ello, sino que incluso, han mejorado su confianza e imagen, junto con los llamados bancos éticos. Por este motivo, la banca cooperativa y la banca ética pueden actuar como motivadores del cambio del negocio bancario hacia un enfoque de RSE, que les permita, no solo mejorar su contribución a la sostenibilidad del territorio, sino también a consolidar su posición empresarial en el mercado.

\section{Referencias bibliográficas}

ACI (1995) Declaración de identidad cooperativa. principios cooperativos. Manchester: Alianza Cooperativa Internacional.

Aker, J.C.; Boumnijel, R.; McClelland, A. y Tierney, N. (2011) Zap it to me: The shortterm impacts of a mobile cash transfer program. Working Paper $\mathrm{N}^{\circ} 263$, Washington DC: Centro para el Desarrollo Mundial.

Banerjee, A.; Duflo, E.; Glennerster, R. y Kinnan, C. (2015) The miracle of microfinance? evidence from a randomized evaluation, American Economic Journal: Applied Economics, Vol. 7, No 1, pp. 22-53.

Belhouari, A.; Buendía Martínez, I.; Lapointe, M. J. y Tremblay, B. (2005) La responsabilidad social de las empresas: ¿Un nuevo valor para las cooperativas?, CiriecEspaña, Revista de Economía Pública, Social y Cooperativa, № 53, pp. 191-208.

Bruhn, M. y Love, I. (2012) The economic impact of expanding access to finance in México. Banking the World: Empirical Foundations of Financial Inclusion. Cambridge: MIT Press Cambridge Mass.

Brune, L.; Giné, X.; Goldberg, J. y Yang, D. (2011) Commitments to save: A field experiment in rural malawi. http://econweb.umd.edu/ goldberg/docs/bggy_mwisavings.pdf.

Buendía Martínez, I. y Tremblay, B. (2015) Responsabilidad social y cooperativismo: Un análisis desde la industria bancaria canadiense, Gizarte Ekonomiaren Euskal AldizkariaRevista Vasca de Economía Social, No 11, pp. 59-74. 
Burgess, R. y Pande, R. (2005) Do rural banks matter? evidence from the Indian social banking experiment, The American Economic Review, Vol. 95, No 3, pp. 780-795.

Carrasco Monteagudo, I. (2005) La ética como eficiencia: la responsabilidad social en las cooperativas de crédito españolas, Ciriec-España, Revista de Economía Pública, Social y Cooperativa, $\mathrm{N}^{\mathrm{o}}$ 53, pp. 351-367.

Carroll, A. (1999) Corporate Social Responsibility, Business \& Society, Vol. 38, № 3, pp. 268-295.

Carroll, A. B. (1979) A three dimensional conceptual model of corporate performance, Academy of Management Review, $\mathrm{N}^{\circ} 184$, pp. 497-505.

Cea Moure, R. (2010) La responsabilidad social corporativa en las entidades bancarias de la unión europea: Análisis empírico y propuesta de modelo normalizado. Madrid: Tesis Doctoral, Universidad Autónoma de Madrid.

CECA (2008) Libro verde sobre la responsabilidad social corporativa en el sector financiero: Una aproximación a la sostenibilidad desde las entidades financieras. Madrid: Confederación Española de Cajas de Ahorros.

Chambers, C. y Day, R. (2009) The Banking Sector and CSR: An Unholy Allian-ce?, Financial Regulation International, Vol. 12, No 9, pp. 13-20.

COMISIÓN EUROPEA (2002) Comunicación de la comisión relativa a la responsabilidad social de las empresas: Una contribución empresarial al desarrollo sostenible. Bruselas: Comisión Europea.

Cortes García, F. J. y Belmonte Ureña, L. J. (2010) La base social de las cooperativas de crédito. la importancia de la responsabilidad social corporativa?, Revista de Estudios Empresariales, $\mathrm{N}^{\mathrm{o}} 2$, pp. 35-53.

Cull, R.; Ehrbeck, T. y Holle, N. (2014) La inclusión financiera y el desarrollo: Pruebas recientes de su impacto. The Consultative Group to Assist the Poor CGAP. http://www.cgap.org/sites/default/files/FocusNoteFinancialInclusionandDeelopmentApr il.

Davis, K. (1967) Understanding the social responsibility puzzle, Business Horizons, Vol. $10, \mathrm{~N}^{\mathrm{o}} 4$, pp. $45-51$.

De La Cuesta González, M. (2006) Responsabilidad del sector bancario. Su contribución a un desarrollo más sostenible, Papeles de Economía Española, № 108, pp. 173-190.

De La Cuesta González, M. (2017) La responsabilidad social en el negocio bancario. Una mirada hacia la inclusión financiera de calidad. En Sanchis Palacio, J. R.; Campos Climent, V. (directores): Responsabilidad Social y ética empresarial en las entidades bancarias, Valencia: Universitat de València; pp. 21-42.

De La Cuesta González, M.; Muñoz Torres, M. J. y Fernández Izquierdo, M. (2006) Analysis of social performance in the Spanish financial industry through public data. A proposal, Journal of Business Ethics, Vol. 69, No 3, pp. 289-304.

Decker, O.S. (2004) Corporate social responsibility and structural change in financial services, Managerial Auditing Journal, Vol. 19, № 6, pp. 712-728.

Donaldson, T. y Dunfee, T.W. (1994) Towards a unified conception of business ethics: Integrative, Academy of Management Review, Vol. 19, No 2, pp. 252-252.

Douglas, A.; Doris, J. y Johnson, B. (2004) Corporate Social Reporting in Irish Financial Institutions. Case Study, The TQM Magazine, Vol. 16, No 6, pp. 387-395.

ELKINGTON, J. (1997) Cannibals with forks: The triple bottom line of the 21st century business. Oxford: Capstone Publishing.

Esteban Sánchez, P.; De La Cuesta González, M. y Paredes Gázquez, J.D. (2017) Corporate Social Performance and its relation with Corporate Financial Performance: international 
evidence in the banking industry, Journal of Cleaner Production, Vol. 162, $\mathrm{N}^{\mathrm{o}} 20$, pp. 1102-1110.

EUROSIF (2016) SRI Study 2016. London: Eurosif. https://www.eurosif.org/sri-study2016/.

Fernández Olit, B.; De La Cuesta González, M. y Pardo, E. (2013) Principales riesgos sociales y ambientales del negocio bancario en tiempos de crisis. Madrid: Cuaderno Red de Cátedras Telefónica, UNED.

Fernández Olit, B. y De La Cuesta González, M. (2014) Evaluación de impactos ambientales y sociales del negocio de banca comercial en Europa durante el periodo 2006-2009, Estudios de Economía Aplicada, Vol. 3, No 2, pp. 567-592.

FMI (2016) Corruption: Costs and mitigating strategies. Staff Discussion Note No. 16/05: International Monetary Fund. Retrieved from Social Science Premium Collection Retrieved from https://search.proquest.com/docview/1846442417.

Freeman, R.E. (1994) The politics of stakeholder theory: Some future directions, Business Ethics Quarterly, Vol. 4, No 4, pp. 409-421.

Fundazioa, E. (2011) Cooperativas y fondos de reserva en perspectiva europea, Gizarte Ekonomiaren Euskal Aldizkaria-Revista Vasca de Economía Social, No 3, pp. 133-143.

García De Los Salmones, M.M.; Herrero, A. y Rodríguez Del Bosque, I. (2005) Influence of corporate social responsibility on loyalty and valuation of services, Journal of Business Ethics, Vol. 61, № 4, pp. 369-385.

García-Torea, N.; Fernández-Feijoo, B. y De La Cuesta González, M. (2017) The influence of ownership structure on the transparency of CSR reporting: empirical evidence of Spain, Spanish Journal of Finance and Accounting, Vol. 46, No 3, pp. 249-271.

Garriga, E. y Melé, D. (2004) Corporate social responsibility theories: Mapping the territory, Journal of Business Ethics, Vol. 53, No 1, pp. 51-71.

Gil, R.B.; Gutiérrez, T.M. y Pérez, J.M.P. (2009) La imagen corporativa de la banca comercial. diferencias entre segmentos de consumidores, Universia Business Review, Vol. 1, No 21, pp. 66-83.

Han, R. y Melecky, M. (2013) Financial inclusion for financial stability: Access to bank deposits and the growth of deposits in the global financial crisis. Washington, DC: Banco Mundial.

Hassan, A. (2009) Corporate Social Responsibility of Islamic Financial Institutions and Businesses, Humanomics, Vol. 25, No 3, pp. 177-188.

Hassan, A. y Syafri, S. (2009) Exploring Corporate Social Responsibility Disclo-sure: The Case of Islamic Banks, International Journal of Islamic and Middle Eastern Finance and Management, Vol. 3, No 3, pp. 203-227.

Jahan, S. y McDonald, B. (2011) A bigger slice of a growing pie-developing the financial sector accelerates economic growth and can enhance income equality, Finance and Development-English Edition, Vol. 48, № 3, pp. 16-20.

Janzen, S.A. y Carter, M.R. (2013) After the drought: The impact of microinsurance on consumption smoothing and asset protection. NBER Working Paper n. ${ }^{\circ}$ 19702, Cambridge, Mass.: National Bureau of Economic Research.

Jensen, M.C. (2002) Value maximization, stakeholder theory, and the corporate objective function, Business Ethics Quarterly, Vol. 12, № 2, pp. 235-256.

Kahn, M.; Halabi, A.K. y Samy, M. (2009) Corporate Social Responsibility (CSR) Reporting: A Study of Selected Banking Companies in Bangladesh, Social Responsibility Journal, Vol. 5, No 3, pp. 344-357. 
Kempson, E.; Whyley, C.; Caskey, J. y Collard, S. (2000) Financial Exclusion: A Literature and Research Review. Consumer Policy and Research. London: Financial Services Authority.

Linnanen, L. y Panapanaan, V.M. (2002) Roadmapping CSR in Finnish companies. Helsinki: Helsinki University of Technology.

Logsdon, J.M. y Wood, D.J. (2002) Business citizenship: From domestic to global level of analysis, Business Ethics Quarterly, Vol. 12, No 2, pp. 155-187.

Maignan, I.; Ferrell, O.C. y Hult, G.T.M. (1999) Corporate citizenship: Cultural antecedents and business benefits, Journal of the Academy of Marketing Science, Vol. $27, \mathrm{~N}^{\mathrm{o}} 4$, pp. 455-469.

Maingot, M. y Zeghal, D. (2008) An Analysis of Corporate Governance Informa-tion Disclosure by Canadian Banks, Corporate Ownership \& Control, Vol. 5, No 2, pp. 225 236.

Malo, M. y Tremblay, B. (2004) Coopératives financieres et solidarité, Finance \& Bien Commun, $\mathrm{N}^{\mathrm{o}} 3$, pp. 66-73.

Marin, L. y Ruiz, S. (2007) I need you too! corporate identity attractiveness for consumers and the role of social responsibility, Journal of Business Ethics, Vol. 71, № 3, pp. 245260.

Martínez García De Leaniz, R.P. (2015) Influencia de la responsabilidad social corporativa en el comportamiento del consumidor turístico: un estudio desde la perspectiva del turismo sostenible. Santander: Tesis Doctoral, Universidad de Cantabria.

McDonald, L. y Rundle-Thiele, S. (2008) Corporate Social Responsibility and Banks Customer Satisfaction: A Research Agenda, International Journal of Bank Marketing, Vol. 26, No 3, pp. 170-182.

Melé, D. (2002) Not only stakeholder interests. The firm oriented toward the common good. Notre Dame: Notre Dame University Press.

Melián Navarro, A.; Sanchis Palacio, J.R. y Soler Tormo, F. (2010) El Crédito Cooperativo como instrumento financiero para el fomento del emprendimiento en tiempos de crisis, Ciriec-España, Revista de Economía Pública, Social y Cooperativa, №68, pp. 111139.

Moneva, J.M. y Lizcano, J.L. (2003) Marco conceptual de la responsabilidad social corporativa. Barcelona: Asociación Española de Contabilidad y Auditoría de Cuentas (AECA). Documento $\mathrm{N}^{\mathrm{o}} 1$.

Mozas Moral, A. y Puentes Poyatos, R. (2010) La responsabilidad social corporativa y su paralelismo con las sociedades cooperativas, REVESCO. Revista de Estudios Cooperativos, Tercer Cuatrimestre, No 103, pp. 75-100.

Muñoz, M.J.; Fernández, M.A. y De Le Cuesta, M. (2004) Responsabilidad social en el sistema bancario: Una primera aproximación de evaluación para el caso español. Papeles de Etica, Economía y Dirección, XII Conferencia Anual de Ética, Economía y Dirección.

OCDE (2002) Guidelines for multinational enterprises. Paris: Organización para la Cooperación y el Desarrollo Económico.

Ontiveros, E. y Sánchez, E. (2014) Cooperativas de crédito, community banking y pymes. Madrid: Unión Nacional de Cooperativas de Crédito.

Orozco, Y.V.D.; Barón, D.M. y Gómez, S.L.B. (2012) Una revisión a la investigación en responsabilidad social corporativa en el sector bancario, Equidad y Desarrollo, $\mathrm{N}^{\circ} 18$, pp. 121-150. 
Palomo Zurdo, R.J. y Valor Martínez, C. (2001) Banca cooperativa: Entorno financiero y proyección social. Madrid: UNACC.

Panwar, R.; Rinne, T.; Hansen, E. y Juslin, H. (2006) Corporate responsibility: Balancing economic, environmental, and social issues in the forest products industry, Forest Products Journal, Vol. 56, No 2, pp. 4-12.

Pérez Ruiz, A. y Rodríguez Del Bosque, I. (2011) La imagen de responsabilidad social corporativa en un contexto de crisis económica: El caso del sector financiero en España, Universia Business Review, Primer trimestre, pp. 14-29.

Pérez Ruiz, A. y Rodríguez Del Bosque, I. (2012) The role of CSR in the corporate identity of banking service providers, Journal of Business Ethics, Vol. 108, № 2, pp. 145-166.

Pérez Ruiz, A. y Rodríguez Del Bosque, I. (2015) Customer responses to the CSR of banking companies, Journal of Product \& Brand Management, Vol. 24, № 5, pp. 481493.

Polonsky, M. y Scott, D. (2005) An Empirical Examination of the Stakeholder Strategy Mix, European Journal of Marketing, Vol. 39, № 9, pp. 1199-1215.

Porter, M.E. y Kramer, M.R. (2002) The competitive advantage of corporate philanthropy, Harvard Business Review, Vol. 80, No 12, pp. 56-68.

Porter, M.E. y Kramer, M.R. (2011) Creating Shared Value. How to reinvent capitalism and unleash a wave of innovation and growth, Harvard Business Review, Vol. 89, ${ }^{\circ}$ 1/2, pp. 2-17.

Puentes Poyatos, R.; Velasco Gámez, M. y Vilar Hernández, J. (2009) El buen gobierno corporativo en las sociedades cooperativas, REVESCO. Revista de estudios cooperativos, Segundo Cuatrimestre, No 98, pp. 118-140.

Reyes, L. E (2008) La investigación en responsabilidad social en los diez últimos años: un análisis de las publicaciones en business and management, XXII Congreso Anual AEDEM: Estableciendo puentes en una economía global, Salamanca.

Rice, T. y Peter, C. (2015) Banking on Corporate Social Responsibility, Journal of Management Sciences, Vol. 2, No 2, pp. 256-273.

Rosero Villabon, O.G. (2015) Relación entre desempleo financiero y responsabilidad social empresarial: Un análisis en la banca europea. Valladolid: Tesis Doctoral Universidad de Valladolid.

Ruiz, M.; Ríos, A. y Tirado, P. (2009) La responsabilidad social y la crisis económica. ¿Responden las entidades financieras españolas a los grupos de interés?, Ciriec-España, Revista de Economía Pública, Social y Cooperativa, No 65, pp. 33-45.

Sanchis Palacio, J.R. y Campos Climent, V. (dirs.) (2017) Responsabilidad social y ética empresarial en las entidades bancarias. Valencia: Publicacions de la Universitat de Valencia.

Sarro Alvarez, M.; Cuesta Valiño, P.C. y Penelas Leguía, A.P. (2007) La Responsabilidad Social Corporativa (RSC). Una Orientación Emergente en la Gestión de las Entidades Bancarias Españolas,. En Ayala, J. C. (Ed.): Conocimiento, Innovación y Emprendedores: Camino al Futuro. Logroño: Universidad de La Rioja, pp. 1928-1942.

Secchi, D. (2007) Utilitarian, managerial, and relational theories of corporate social responsibility, International Journal of Management Reviews, Vol. 9, No 4, pp. 347373.

Seguí, A. y Palomero, S. (2013) The Spanish Banks in face of the Corporate Social Responsibility Standards: previous analysis of the financial crisis, Revista Brasileira de Gestao de Negócios -Review of Business Management, Vol. 15, № 49, pp. 562-581. 
Senthikumar, N.; Ananth, A. y Arulraj, A. (2011) Impact of Corporate Social Responsibility on Customer Satisfaction in Banking Service, African Journal of Business Management, Vol. 5, No 7, pp. 3028-3039.

Server Izquierdo, R.J. y Capó Vicedo, J. (2009) La responsabilidad social empresarial en un contexto de crisis. repercusión en las sociedades cooperativas, Ciriec-España, Revista de Economía Pública, Social y Cooperativa, No 65, pp. 7-31.

Server Izquierdo, R.J. y Villalonga Grañana, I. (2008) Responsabilidad social y cooperativismo. el fondo de educación y promoción (FEP) como indicador social en el caso del cooperativismo de crédito, Estudios de Economía Aplicada, Vol. 26, № 1, pp. 133-157.

Soler Tormo, F. y Melián Navarro, A. (2012) Cooperativas de crédito y Banca social. Viejas y nuevas respuestas éticas y solidarias a problemas de siempre. REVESCO. Revista de Estudios Cooperativos, Tercer Cuatrimestre, $\mathrm{n}^{\circ}$ 109, pp. 45-80. DOI: 10.5209/rev_REVE.2012.v109.40655.

Truño i Gual, J. (2016) La responsabilidad social corporativa: Una aproximación al sector bancario. Barcelona: Tesis Doctoral Universitat Autónoma de Barcelona.

Turker, D. (2009) Measuring corporate social responsibility: A scale development study, Journal of Business Ethics, Vol. 85, No 4, pp. 411-420.

UNITED NATIONS (2000) Resolution 547123, adopted by general assembly, cooperatives in social development. Document A/RES/54/123. Washington: United Nations.

Van Marrewijk, M. (2003) Concepts and definitions of CSR and corporate sustainability: Between agency and communion, Journal of Business Ethics, Vol. 44, No 2, pp. 95-105.

Vargas Sánchez, A. y Vaca Acosta, R.M. (2005) Responsabilidad social corporativa y cooperativismo: Vínculos y potencialidades, Ciriec-España, Revista de Economía Pública, Social y Cooperativa, No 53, pp. 241-260. 